\title{
Joint Power Allocation for the Multi-User NOMA-Downlink in a Power-Line-Fed VLC Network
}

\author{
Simeng Feng, Tong Bai, Lajos Hanzo, Fellow, IEEE
}

\begin{abstract}
Visible light communications (VLC) constitutes as a promising downlink technique of supporting the ever-increasing tele-traffic. Since the ubiquitous mains power line constitutes a natural backbone feed for VLC, a combined power line and VLC network is conceived, where the source data is fed by power line communications (PLC) into the VLC network. We refer to it as a PLC-VLC network, where the power-line connects the light emitting diodes (LED) without requiring a duplicated filter-backbone. Then the information is forwarded to multiple users via visible light. Furthermore, to increase the downlink bit rate, non-orthogonal multiple access (NOMA) is invoked in VLC. For the sake of maximizing the sum-throxughput, we conceive a joint PLC-VLC power allocation (JPA) strategy, where the power allocated both to the PLC and to the VLC links is jointly determined upon analysing the power-relationship between them. Our simulations demonstrate that NOMA significantly outperforms orthogonal multiple access (OMA) with the aid of the proposed JPA.
\end{abstract}

Index Terms-Visible light communications (VLC), power line communications (PLC), non-orthogonal multiple access (NOMA).

\section{INTRODUCTION}

As a benefit of its inherent advantages, such as its licensefree spectrum and strong immunity to electromagnetic interference, light emitting diode (LED) based visible light communication (VLC) has been considered as an appealing complementary technology to radio-frequency (RF) communiation [1]. The ubiquitous mains wiring constitutes a natural choice for feeding the LEDs [2]. However, power line communication (PLC) constitutes a hostile medium due to the impulsive noise and multipath effects [3]. Fortunately, sophisticated OFDMbased PLC is capable of mitigating these effects in highspeed transmission [4]. Hence a combined PLC-VLC network is conceived as an economical candidate for smart-home networking.

With the emergence of the Internet of Everything (IoE), a further explosive growth of the tele-traffic is expected. In contrast to conventional orthogonal multiple access (OMA), the non-orthogonal multiple access (NOMA), allows multiple users to share a given time-or-frequency-slot by multiplexing them in the power-domain [5]. Although the concept was intended for RF systems, it is also beneficial for achieving

L. Hanzo would like to acknowledge the financial support of the Engineering and Physical Sciences Research Council projects EP/Noo4558/1, EP/PO34284/1, COALESCE, of the Royal Society's Global Challenges Research Fund Grant as well as of the European Research Council's Advanced Fellow Grant QuantCom.

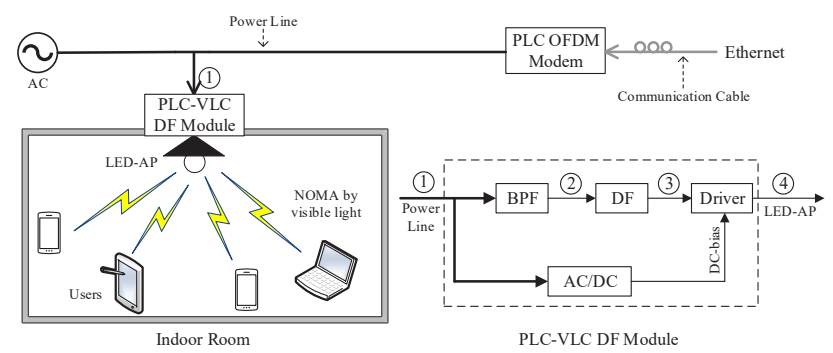

Fig. 1: Schematic of the PLC-VLC network with an LED-AP and multiple users, where the classic OFDM scheme is adopted in the PLC link, while the power-domain NOMA VLC downlink serves multiple users.

a high data-rate in VLC systems, since the off-the-shelf LEDs have limited bandwidth [6]. Given the different communication environment of RF and VLC, the potential benefits of adopting NOMA in the VLC downlink are in the following aspects: (i) Due to its LoS-dominated propagation nature, the VLC system has an inherently high SNR, which is particularly beneficial, when NOMA is adopted [7]; (ii) Since VLC is typically used in indoor communication, the number of users supported by a single LED based access point (LED-AP) is lower than that of the RF system, which allows the successive interference cancellater (SIC) to perform well [8]; (iii) As a benefit of the predominantly static VLC channel, the channel state information (CSI) can be estimated relatively accurately [9], which is important for the subsequent power allocation of NOMA. Therefore, in this paper, we aim for exploring the sum-throughput attained by a PLC-VLC network in a multi-user scenario, where OFDM is employed in PLC and NOMA is invoked in the VLC downlink. Since PLC acts as the backhaul of VLC, the throughput is jointly determined by them. Although the higher the throughput provided by PLC, the higher the throughput attained by VLC, but naturally, an increased power is required by PLC for increasing its throughput. To handle the dependent relationship between PLC and VLC, instead of separately exploring both networks, a novel joint power allocation (JPA) strategy is proposed for our PLC-VLC network. To expound further, the proposed JPA strategy investigates the power relationship of OFDM-based PLC and NOMA-aided VLC, while the sum-throughput is maximized upon jointly determining the power allocation for each subcarrier in PLC and for the multiple users in VLC, while satisfying the transmission quality required.

The rest of the paper is organized as follows. Our PLC-VLC 
system model is presented in Section II. The constrained sumthroughput maximization problem is formulated in Section III, which is solved by the proposed JPA in Section IV. Our results are discussed in Section V, whilst our conclusions are drawn in Section VI.

\section{System Model}

In an indoor scenario, we assume that an LED-AP faces downward providing access to $M$ users, where the power line has already been installed for power supply. As seen in Fig. 1 , the source data arriving from the Ethernet is fed into the power line through a PLC OFDM modem. Since the PLC link is used as the backhaul in our proposed PLC-VLC network, we assume that the information of multiple users is transmitted as a serial bit stream via PLC. The mapping strategy is known at both the PLC OFDM modem and the PLC-VLC DF module, so that the information destined for different users can be distinguished by the VLC link. However, the study of the mapping strategy is beyond the scope of this paper. The OFDM signal propagating along with the alternating current $(\mathrm{AC})$ at $50 \mathrm{~Hz}$ (as shown at (1) is received by the PLC-VLC decodeand-forward (DF) module. Since the frequency of the OFDM signal is much higher than $50 \mathrm{~Hz}$, a bandpass filter (BPF) is employed to extract the PLC signal (as shown at (2)). The signal at (2) is then demodulated and decoded by the DF unit, where the decoded bit stream is then remodulated for the VLC optical wireless (OW) link, as shown at (3) of Fig. 1. Since the LED-AP is driven by direct current (DC), a DC-bias, obtained by an AC/DC converter ${ }^{1}$, is added to the signal at (3), so that the OW signal suitable for LED transmission is obtained at (4). Note that the network is monitored by a central controller, which is responsible for the power allocation (PA) relying on the feedback information received from the users via the Wi-Fi (RF) uplink.

\section{A. OFDM-Based Power Line Transmission}

Generally, the PLC channel can be modelled by two approaches, namely the bottom-up and the top-down. In this paper, the transfer function $g(f)$ of the PLC channel based on the top-down approach is invoked by relying on the parameters measured in [11], as shown in Fig. 2. Since OFDM is employed in PLC for counteracting the frequency-selective channel and its high-noise environment, the channel gain $g_{n}$ at the $n$th subcarrier is obtained by averaging the function $g(f)$ over the surrounding $N$ subcarriers and plotting the average at each band's central frequency. To elaborate, the main noise source in PLC is the colored background noise, which has a variance of $\sigma_{\mathrm{p}, n}^{2}$ at the $n$th subcarrier [12]. Another impairment in PLC is the impulsive disturbance, which is non-stationary and usually occurs with a relatively low probability [2]. For simplicity, we denote the probability of the PLC channel being

\footnotetext{
${ }^{1}$ There are numerous AC/DC converter designs, but the classic bridge rectifier is perhaps the one most commonly employed [10]. To expound further, the bridge rectifier consists of four diodes and a capacitor. By exploiting the specific characteristics of the diode and capacitor, the bipolar input AC signal can be converted to a near-constant amplitude.
}

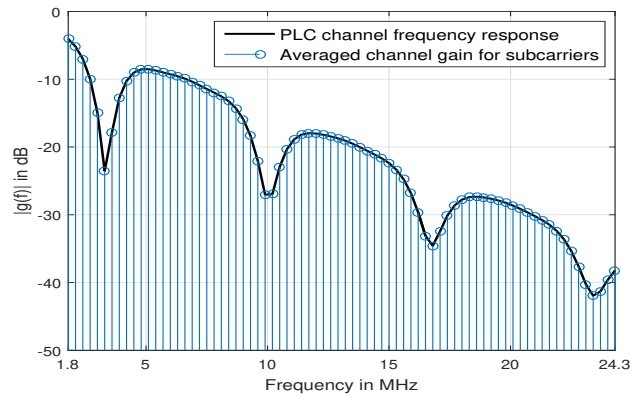

Fig. 2: Frequency response of PLC channel modelled in [11], where the channel gain for OFDM-subcarriers is obtained by averaging $g(f)$ at their centre frequencies.

free from impulsive noise by $\varrho$ [13]. Hence, the throughput of the OFDM-based PLC may be formulated as:

$$
R_{\mathrm{plc}}=\varrho \sum_{n=1}^{N} B \log _{2}\left(1+\lambda_{n} p_{\mathrm{plc}, n}\right),
$$

where $N$ is the number of subcarriers, $B=B_{\mathrm{p}} / N$ is the bandwidth of each subcarrier, while the bandwidth of PLC is denoted by $B_{\mathrm{p}}$. The power allocated to the $n$th subcarrier is denoted by $p_{\mathrm{plc}, n}$, where we have $\lambda_{n}=\left|g_{n}\right|^{2} / \sigma_{\mathrm{p}, n}^{2}$.

\section{B. NOMA Aided VLC Downlinks}

Since the VLC channel can be modelled by a single-tap AWGN channel and the energy of its reflections decreases rapidly, a Lambertian model is considered for user $m$ with respect to the line-of-sight $(\mathrm{LoS})$ and the first reflected path within its field-of-view (FoV) as ${ }^{2}$ [14]:

$$
\begin{aligned}
& h_{\mathrm{LoS}, m}= \frac{(\alpha+1) A_{p}}{2 \pi D_{m}^{2}} \cos ^{\alpha}(\phi) \cos (\psi) g_{o f}(\psi) g_{o c}(\psi), \\
& h_{\mathrm{Ref}, m}=\int_{\text {walls }} \frac{\rho(\alpha+1) A_{p}}{2 \pi^{2} D_{a, r}^{2} D_{r, m}^{2}} \cos ^{\alpha}(\phi) \cos (\psi) \\
& \cos \left(w_{1}\right) \cos \left(w_{2}\right) g_{o f(\psi)} g_{o c}(\psi) d A_{\text {wall }} .
\end{aligned}
$$

Hence, the aggregated VLC channel of user $m$ is obtained as $h_{m}=h_{\mathrm{LoS}, m}+h_{\mathrm{Ref}, m}$. Without loss of generality, the users are sorted in ascending order based on their channel gains, according to $\left|h_{1}\right|^{2} \leq \cdots \leq\left|h_{M}\right|^{2}$. For supporting multi-user access, the power-domain NOMA principle is invoked, where the transmitted signal of the LED-AP is $\sum_{m=1}^{M} s_{m} \sqrt{p_{\mathrm{vlc}, m}}$, while $s_{m}$ is the signal intended for user $m$ and $p_{\mathrm{vlc}, m}$ is its allocated power. According to the NOMA regime, the received VLC signal at user $m$ is expressed as:

$$
y_{m}=h_{m} \sum_{l=1}^{M} s_{l} \sqrt{p_{\mathrm{vlc}, l}}+n_{m},
$$

${ }^{2}$ We have $\alpha$ and $\rho$ as the order of Lambertian emission and reflection coefficient, while $A_{p}$ is the physical area of the photo-diode. Also, $D_{m}$ is the distance between user $m$ and the AP, where $D_{a, r}$ and $D_{r, m}$ is the distance between the AP and a reflection point, and that between the reflection point and user $m$, respectively. The angle of irradiance and of incidence are $\phi$ and $\psi$, respectively. The angles of irradiance to the reflection point and to the user are $w_{1}$ and $w_{2}$, while $g_{o f}(\psi)$ and $g_{o c}(\psi)$ is the gain of the optical filter and of the concentrator, respectively. 
where $n_{m}$ denotes the AWGN of user $m$ with variance $\sigma_{\mathrm{v}}^{2}$. In SIC, the higher-power user is detected first, because it is affected by the interference imposed by the lower-power user to a lesser extent. Then its detected signal is remodulated and subtracted from the composite signal, hence decontaminating it. Therefore, the interference imposed on user $m$ only comes from the signal destined for user $(m+1)$ to $M$. These SIC operations continue until we arrive at the signal of the lowest-power user. Then the achievable throughput ${ }^{3}$ of user $m$ becomes:

$$
R_{\mathrm{vlc}, m}=\frac{B_{\mathrm{v}}}{2} \log _{2}\left(1+\frac{\left|h_{m}\right|^{2} p_{\mathrm{vlc}, m}}{\left|h_{m}\right|^{2} \sum_{l=m+1}^{M} p_{\mathrm{vlc}, l}+\sigma_{\mathrm{v}}^{2}}\right),
$$

where $B_{\mathrm{v}}$ is the bandwidth of VLC. Due to the intensity modulation with direct detection (IM/DD) of VLC system [15], a scaling factor of $1 / 2$ is applied for taking into account the throughput reduction imposed by the implementation of Hermitian symmetry to obtain real-valued signals [16]. Upon defining $P_{\mathrm{vlc}, m}=\sum_{l=m}^{M} p_{\mathrm{vlc}, l}$, Eq. (5) can be rewritten as:

$$
R_{\mathrm{vlc}, m}=\frac{B_{\mathrm{v}}}{2} \log _{2}\left(\frac{\eta_{m} P_{\mathrm{vlc}, m}+1}{\eta_{m} P_{\mathrm{vlc}, m+1}+1}\right),
$$

where $\eta_{m}=\left|h_{m}\right|^{2} / \sigma_{\mathrm{v}}^{2}$. Note that we have $P_{\mathrm{vlc}, M+1}=0$.

\section{Constrained Problem Formulation}

In the PLC-VLC network conceived, our objective is to maximize the sum-throughput attained by the $M$ users upon jointly considering the data-carrying power allocation of both the PLC and VLC component ${ }^{4}$. Specifically, the total transmit power $P_{T}$ is restricted in reality, which has to be carefully assigned to the individual OFDM-subcarriers in PLC and to the multiple VLC users, respectively. Furthermore, since PLC is invoked as the backhaul of VLC, the achievable sum-throughput of the PLC-VLC network is limited by the performance of PLC. To maintain fairness, the minimum rate requirement $r_{m}$ of each user has to be satisfied. Mathematically, our constrained throughput-maximization problem is hence formulated as:

$$
\begin{array}{ll} 
& \max _{\boldsymbol{p}_{\mathrm{plc}}, \boldsymbol{p}_{\mathrm{vlc}}} \sum_{m=1}^{M} R_{\mathrm{vlc}, m} \\
\text { s.t. } & P_{\mathrm{plc}}+P_{\mathrm{vlc}, 1} \leq P_{T} ; \\
& \sum_{m=1}^{M} R_{\mathrm{vlc}, m} \leq R_{\mathrm{plc}} ; \\
& R_{\mathrm{vlc}, m} \geq r_{m}, \quad \forall m ; \\
& \boldsymbol{p}_{\mathrm{plc}} \geq \mathbf{0}_{N \times 1}, \quad \boldsymbol{p}_{\mathrm{vlc}} \geq \mathbf{0}_{M \times 1},
\end{array}
$$

where we have $P_{\mathrm{plc}}=\sum_{n} p_{\mathrm{plc}, n}, \boldsymbol{p}_{\mathrm{plc}}=\left[p_{\mathrm{plc}, 1}, \cdots, p_{\mathrm{plc}, N}\right]^{T}$ and $\boldsymbol{p}_{\mathrm{vlc}}=\left[p_{\mathrm{vlc}, 1}, \cdots, p_{\mathrm{vlc}, M}\right]^{T} \cdot \mathbf{0}_{N \times 1}$ represents a $N$ dimen-

\footnotetext{
${ }^{3}$ In this contribution, we assume that perfect SIC can be performed in our NOMA aided VLC downlink. Explicitly, the interference imposed on user $m$ by all the users having indices of 1 to $m-1$, can be perfectly decoded without errors.

${ }^{4}$ We note that although data-carrying PLC power is only a small fraction of the illumination power, it is paramount to keep it as low as possible for avoiding the contamination of the PLC network.
}

sional column zero vector and so does $0_{M \times 1}$. Note that the feasibility of problem (7) is considered in Theorem 1.

Theorem 1. There exists at least one feasible solution of (7) if and only if the following condition is satisfied:

$$
\begin{aligned}
& \sum_{n=1}^{N}\left[2^{\left(\sum_{m} r_{m}-\varrho B \sum_{n} \log _{2} \lambda_{n}\right) / \varrho B_{p}}-\frac{1}{\lambda_{n}}\right]^{+} \\
& +\sum_{m=1}^{M} 2^{\sum_{j=1}^{m-1} 2 r_{j} / B_{v}}\left(2^{2 r_{m} / B_{v}}-1\right) / \eta_{m} \leq P_{T} .
\end{aligned}
$$

Proof. Please see Appendix A.

Theorem 1 indicates that problem (7) is tractable when $P_{T}$ meets the feasibility condition of (8). Therefore, the total available power $P_{T}$ has to be higher than the minimum power required by the PLC-VLC network so that the minimum rate requirement of each user can be satisfied.

\section{Joint Power Allocation}

When $P_{T}$ satisfies the feasibility condition revealed in Theorem 1, upon analysing the relationship between the PLC as well as VLC links, we will demonstrate that the total power required by VLC is a function of $P_{\text {plc }}$. Therefore, with the aid of (7b), the power allocated to PLC and to VLC can be jointly decided. To elaborate, the Lagrangian function of (7) is given by:

$$
\begin{aligned}
& \mathcal{L}\left(\boldsymbol{p}_{\mathrm{plc}}, \boldsymbol{p}_{\mathrm{vlc}}, \alpha, \beta, \boldsymbol{\mu}\right)=\sum_{m=1}^{M} \frac{B_{\mathrm{v}}}{2} \log _{2}\left(\frac{\eta_{m} P_{\mathrm{vlc}, m}+1}{\eta_{m} P_{\mathrm{vlc}, m+1}+1}\right) \\
& +\alpha\left(P_{T}-P_{\mathrm{plc}}-P_{\mathrm{vlc}, 1}\right)+\beta\left[\varrho B \sum_{n=1}^{N} \log _{2}\left(1+\lambda_{n} p_{\mathrm{plc}, n}\right)\right. \\
& \left.-\sum_{m=1}^{M} \frac{B_{\mathrm{v}}}{2} \log _{2}\left(\frac{\eta_{m} P_{\mathrm{vlc}, m}+1}{\eta_{m} P_{\mathrm{vlc}, m+1}+1}\right)\right] \\
& +\sum_{m=1}^{M} \mu_{m}\left[\frac{B_{\mathrm{v}}}{2} \log _{2}\left(\frac{\eta_{m} P_{\mathrm{vlc}, m}+1}{\eta_{m} P_{\mathrm{vlc}, m+1}+1}\right)-r_{m}\right]
\end{aligned}
$$

where $\alpha, \beta$ and $\boldsymbol{\mu}=\left[\mu_{1}, \cdots, \mu_{M}\right]^{T}$ are the Lagrange multipliers associated with the (7b) - (7d). The Karush-KuhnTucker (KKT) conditions of (7) are listed as follows:

$$
\begin{aligned}
& \frac{\partial \mathcal{L}}{\partial p_{\mathrm{plc}, n}}=-\alpha+\frac{\varrho \beta B \lambda_{n}}{\ln 2\left(1+\lambda_{n} p_{\mathrm{plc}, n}\right)}=0, \quad \forall n, \\
& \frac{\partial \mathcal{L}}{\partial p_{\mathrm{vlc}, m}}=-\alpha+\frac{B_{\mathrm{v}}}{2 \ln 2}\left\{\frac{\eta_{1}\left(1-\beta+\mu_{1}\right)}{\eta_{1} P_{\mathrm{vlc}, 1}+1}+\right. \\
& \left.\sum_{l=2}^{m}\left[\frac{\eta_{l}\left(1-\beta+\mu_{l}\right)}{\eta_{l} P_{\mathrm{vlc}, l}+1}-\frac{\eta_{l-1}\left(1-\beta+\mu_{l-1}\right)}{\eta_{l-1} P_{\mathrm{vlc}, l}+1}\right]\right\}, \forall m, \\
& \alpha\left(P_{T}-P_{\mathrm{plc}}-P_{\mathrm{vlc}, 1}\right)=0, \\
& \beta\left(R_{\mathrm{plc}}-\sum_{m=1}^{M} R_{\mathrm{vlc}, m}\right)=0, \\
& \mu_{m}\left(R_{\mathrm{vlc}, m}-r_{m}\right)=0, \quad \forall m, \\
& \alpha \geq 0, \beta \geq 0, \boldsymbol{\mu} \geq \mathbf{0}_{M \times 1},(7 b),(7 c),(7 d), \quad(7 e) .
\end{aligned}
$$


Considering $m=1$ from (9b), we have $\alpha=$ $\frac{B_{\mathrm{v}} \eta_{1}\left(1-\beta+\mu_{1}\right)}{2 \ln 2 \times\left(\eta_{1} P_{\mathrm{vlc}, 1}+1\right)}$, which can be further combined with (9a) to get:

$$
\alpha=\frac{1+\mu_{1}}{\ln 2 \times\left[\frac{2\left(\eta_{1} P_{\mathrm{vlc}, 1}+1\right)}{B_{\mathrm{v}} \eta_{1}}+\frac{\lambda_{n} p_{\mathrm{plc}, n}+1}{\varrho B \lambda_{n}}\right]} .
$$

Observe that the denominator of (10) is always positive, regardless of the PA. Furthermore, because we have $\mu_{1} \geq 0$ based on (9f), it can be inferred that $\alpha \neq 0$. Upon recalling (9c), we may conclude that the equality holds only, when $P_{\mathrm{plc}}+P_{\mathrm{vlc}, 1}=P_{T}$. Since (9a) implies that similarly to $\alpha, \beta$ is also positive, it may be readily seen that due to (9d) the sumthroughput achieved by VLC equals to the total throughput of PLC. Furthermore, by exploiting (9b), we arrive at:

$$
\begin{aligned}
& \frac{\partial \mathcal{L}}{\partial p_{\mathrm{vlc}, m}}-\frac{\partial \mathcal{L}}{\partial p_{\mathrm{vlc}, m-1}}=0 \\
& =\frac{B_{\mathrm{v}}}{2 \ln 2}\left[\frac{1-\beta+\mu_{m}}{P_{\mathrm{vlc}, m}+1 / \eta_{m}}-\frac{1-\beta+\mu_{m-1}}{P_{\mathrm{vlc}, m}+1 / \eta_{m-1}}\right] .
\end{aligned}
$$

Upon taking into account that $\left|h_{m-1}\right|^{2}<\left|h_{m}\right|^{2}$, 5 we have $P_{\text {vlc }, m}+1 / \eta_{m}<P_{\text {vlc }, m}+1 / \eta_{m-1}$. It can hence be concluded that (11) equals to 0 if and only if $\mu_{m-1}>\mu_{m}$, leading to $\mu_{1}>\mu_{2}>\cdots>\mu_{M} \geq 0$. Upon considering (9e), we may infer that for user $m \in\{1, \cdots, M-1\}$, the JPA solution is obtained, when his/her achievable throughput is $r_{m}$. To elaborate, provided that $\mu_{M}>0$, the throughput-maximization problem of (7) is readily solved with the achievable VLC sum-throughput given by $\sum_{m=1}^{M} r_{m}$. The PA of both VLC and PLC can thus be readily configured. By contrast, if we have $\mu_{M}=0$, the achievable throughput of user $M$ is $R_{\mathrm{vlc}, M}=R_{\mathrm{plc}}-\sum_{m=1}^{M-1} r_{m}$. Therefore, the expression of $P_{\mathrm{vlc}, M}$ can be obtained according to (6). Relying on $P_{\mathrm{vlc}, M}$, the power $P_{\mathrm{vlc}, M-1}$ can also be obtained with the relationship of $R_{\mathrm{vlc}, M-1}=r_{M-1}$. By recursively reducing the user-index, the general expression of $P_{\mathrm{vlc}, m}$ may be formulated as:

$$
\begin{aligned}
P_{\mathrm{vlc}, m}= & \frac{2^{2\left(R_{\mathrm{plc}}-\sum_{l=1}^{m-1} r_{l}\right) / B_{\mathrm{v}}}}{\eta_{M}}-\frac{1}{\eta_{m}} \\
& -\sum_{i=m}^{M-1} \frac{\left(\eta_{i}-\eta_{i+1}\right) 2^{\sum_{l=m}^{i} 2 r_{l} / B_{\mathrm{v}}}}{\eta_{i} \eta_{i+1}} .
\end{aligned}
$$

For a given PLC power, the maximum rate $R_{\mathrm{plc}}$ is realized when the PA is based on the classic water-filling strategy. Therefore, the achievable throughput of PLC is given by $R_{\text {plc }}=\varrho B \sum_{n} \log _{2}\left[\lambda_{n}\left(P_{\text {plc }}+\sum_{n} 1 / \lambda_{n}\right) / N\right]$, where the power allocated to the $n$th subcarrier is $p_{\text {plc }, n}=$ $\left(\frac{1}{N}\left(P_{\text {plc }}+\sum_{m} \frac{1}{\lambda_{n}}\right)-\frac{1}{\lambda_{n}}\right)^{+}$, where $(x)^{+}=\max \{0, x\}$. Recall the earlier conclusion that the sum power allocated to both VLC and PLC equals to $P_{T}$, where $P_{T}$ is set to be higher than the minimum power required by the PLC-VLC network, as we indicated in Theorem 1. Hence the following function can be formulated:

$$
\Psi=P_{\mathrm{plc}}+P_{\mathrm{vlc}, 1}-P_{T}
$$

${ }^{5}$ If we have $\left|h_{m-1}\right|^{2}=\left|h_{m}\right|^{2}$, a virtual user $m^{\prime}$ is invoked with $r_{m^{\prime}}=r_{m-1}+r_{m}$. Then the JPA is process for user $1, \cdots, m-2, m^{\prime}, m+$ $1, \cdots, M$.

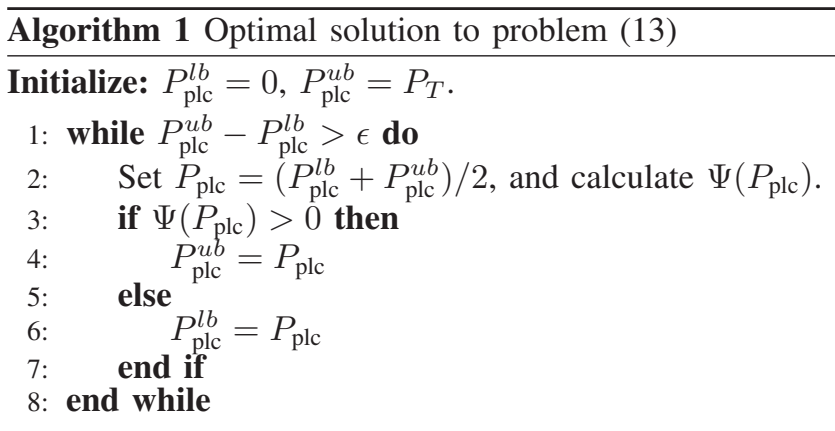

Proposition 1: The function (13) is monotonically increasing when we have $0 \leq P_{\text {plc }} \leq P_{T}$. Furthermore, we have $\Psi(0)<0$ and $\Psi\left(P_{T}\right)>0$

Proof. Upon substituting (12) into (13), it can be found that the function only relies on a single variable, which is $P_{\text {plc }}$. Therefore, by taking the derivative of $\Psi\left(P_{\mathrm{plc}}\right)$ with respect to $P_{\text {plc }}$, we have:

$$
\Psi^{\prime}\left(P_{\mathrm{plc}}\right)=1+\frac{2 \varrho B N 2^{2 R_{\mathrm{plc}} / B_{\mathrm{v}}}}{\eta_{M} B_{\mathrm{v}}\left(P_{\mathrm{plc}}+\sum_{n} 1 / \lambda_{n}\right)}
$$

Observe (14) that we have $\Psi^{\prime}\left(P_{\mathrm{plc}}\right)>0$ when $0 \leq P_{\mathrm{plc}} \leq P_{T}$. Therefore, it can be concluded that the function $\Psi\left(P_{\mathrm{plc}}\right)$ is monotonically increasing within domain $P_{\mathrm{plc}} \in\left[0, P_{T}\right]$. Moreover, when we have $P_{\mathrm{plc}}=P_{T}$, the function of (13) can be written as:

$$
\begin{aligned}
\Psi\left(P_{T}\right)= & \frac{2^{2 R_{\mathrm{plc}} / B_{\mathrm{v}}}-2^{\sum_{l=1}^{M-1} 2 r_{l} / B_{\mathrm{v}}}}{\eta_{M}}+\frac{2^{\sum_{l=1}^{M-1} 2 r_{l} / B_{\mathrm{v}}}-2^{\sum_{l=1}^{M-2} 2 r_{l} / B_{\mathrm{v}}}}{\eta_{M-1}} \\
& +\cdots+\frac{2^{2 r_{1} / B_{\mathrm{v}}}-1}{\eta_{1}} .
\end{aligned}
$$

Recall constraints (7c) and (7d) that we have $R_{\mathrm{plc}} \geq$ $\sum_{m=1}^{M-1} r_{m}$. Therefore, we observe that each term in (15) is positive, leading to $\Psi\left(P_{T}\right)>0$. Furthermore, when we have $P_{\text {plc }}=0$, the function of (13) is expressed as $\Psi(0)=$ $P_{\text {vlc }, 1}-P_{T}$. According to the constraint (7b), it can be easily inferred that $\Psi(0)<0$.

Since the function $\Psi\left(P_{\mathrm{plc}}\right)$ is monotonically increasing, a classic bisection method may be invoked to find the unique value of $P_{\mathrm{plc}}$ with respect to $\Psi\left(P_{\mathrm{plc}}\right)=0$. The bisection searching procedure of obtaining the optimal power of PLC network is formally described in Algorithm 1. Once the optimal power ${ }^{6} P_{\text {plc }}^{*}$ allocated to PLC is obtained by Algorithm 1, the power values of $P_{\mathrm{vlc}, m}^{*}, \forall m$ can be jointly acquired based on (12). Recall the definition of $P_{\mathrm{vlc}, m}=\sum_{l=m}^{M} p_{\mathrm{vlc}, l}$, the power allocated to each VLC user is given by:

$$
p_{\mathrm{vlc}, m}^{*}= \begin{cases}P_{\mathrm{vlc}, m}^{*}-P_{\mathrm{vlc}, m+1}^{*}, & \text { if } m=1, \cdots, M-1 \\ P_{\mathrm{vlc}, m}^{*}, & \text { if } m=M .\end{cases}
$$

\footnotetext{
${ }^{6}$ In this paper, the superscript $\left({ }^{*}\right)$ denotes the obtained optimal result.
} 
TABLE I: List of Parameters

\begin{tabular}{|l|l|}
\hline \multicolumn{2}{|c|}{ I-1. Environment-related Parameters } \\
\hline room size & $4 \times 4 \times 3 \mathrm{~m}^{3}$ \\
AP height & $2.5 \mathrm{~m}$ \\
UE height & $0.85 \mathrm{~m}$ \\
number of UE $M$ & 5 \\
\hline \multicolumn{2}{|c|}{ I-2. VLC-related Parameters } \\
\hline semi-angle at half-illumination $\phi_{1 / 2}$ & $60^{\circ}$ \\
FoV $\Psi_{\text {FoV }}$ & $120^{\circ}$ \\
gain of optical filter $g_{o f}(\psi)$ & 1 \\
gain of optical concentrator $g_{o c}(\psi)$ & 1 \\
physical area for a PD receiver $S$ & $1 \mathrm{~cm}{ }^{2}$ \\
reflection efficiency $\rho$ & 0.75 \\
bandwidth $B_{\mathrm{v}}$ & $20 \mathrm{MHz}$ \\
\hline \multicolumn{2}{|c|}{ I-3. PLC-related Parameters } \\
\hline number of subcarrier $N$ & 1024 \\
bandwidth per subcarrier $B$ & $24.41 \mathrm{KHz}$ \\
impulsive-noise-free probability $\varrho$ & 0.98 \\
\hline \multicolumn{2}{|c|}{ I-4. Power-allocation-related Parameters } \\
\hline bisection accuracy $\epsilon$ & $10^{-4}$ \\
transmit power constraint $P_{T}$ & $5 \mathrm{~W}$ \\
minimum rate requirement $r_{m}$ & $12 \mathrm{Mbits} / \mathrm{s}$ \\
\hline
\end{tabular}

Therefore, the throughput-maximization problem of (7) is solved by our JPA strategy.

Remark: The number of iterations required by Algorithm 1 to satisfy the desired accuracy $\epsilon$ is $\left(\log _{2}\left(P_{T} / \epsilon\right)\right)$. Within each iteration, the complexity of calculating $\Psi\left(P_{\mathrm{plc}}\right)$ is on the order of $\mathcal{O}\left(N+M^{2}\right)$, based on equation of (12). Therefore, the complexity of our proposed JPA is $\mathcal{O}\left[\left(N+M^{2}\right) \log _{2}\left(P_{T} / \epsilon\right)\right]$.

\section{PERformance EVAluation}

In this section, simulations are conducted to investigate the performance of the proposed JPA designed for our PLC-VLC network, where a $4 \times 4 \times 3 \mathrm{~m}^{3}$ indoor room is considered. Note that our simulation results are averaged over hundreds of independent user distributions, where the location of users is randomly generated. The rest of the simulation-related parameters are summarized in Table I.

Figure 3 shows the sum-throughput of our PLC-VLC network under various minimum rate requirements and user densities. As seen in the top subfigure of Fig. 3, a consistent throughput gain can be achieved by the NOMA scheme, while TDMA/OMA falters under strict minimum rate requirements. The superiority of NOMA can also be observed under various user densities, as shown in the second subfigure of Fig. 3. Since NOMA allows multiple users to be supported simultaneously, an increased throughput gain is attained upon increasing the user population, which is an explicit benefit of NOMA over TDMA/OMA. Furthermore, regardless of the specific PA strategy, the sum-throughput attained by NOMA tends to be slightly reduced upon increasing the minimum rate requirement of each user. This is because increasing the minimum rate requirement of each user resluts in allocating more power to the specific users having worse channels, hence leading to a power reduction for PLC.
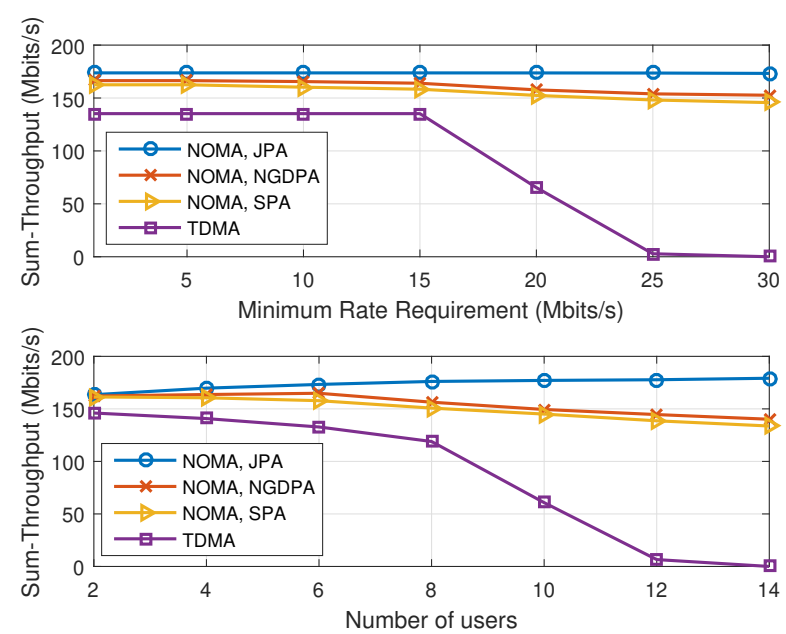

Fig. 3: Sum-throughput v.s. minimum rate requirements and user densities, where the number of users for the first subfigure is 5 and the minimum rate requirement for the second subfigure is $12 \mathrm{Mbits} / \mathrm{s}$.

We also observe that the sum-throughput attained by our proposed JPA is higher than that of the Static PA (SPA) [5] and that of the Normalized Gain Difference PA (NGDPA) [17] benchmarks. It is interesting to observe that the sumthroughput of Fig. 3 acquired by JPA first slightly increases upon increasing the user density and then decreases, while that of the other two PA strategies monotonically decreases. Since JPA succeeds in maintaining the required minimum rate even for those users who do not have the highest channel gain, it encourages the users having the highest channel gain to aim for the highest possible throughput within the affordable power budget.

\section{CONCLUSIONS}

In this paper, we explored a multi-user PLC-VLC downlink scenario. A constrained throughput maximization problem was formulated, which was solved by the proposed JPA. Our simulations demonstrated that the NOMA technique was capable of attaining a higher throughput than OMA. Furthermore, we also demonstrated that the proposed JPA outperformed the previously published SPA and NGDPA benchmarks [5], [17], under diverse minimum rate requirements and user densities. Naturally, some open challenges arise in the proposed PLCVLC network, where the subcarrier allocation for multiple users may have to be considered jointly with the power allocation.

\section{APPENDIX A}

Proof. To prove Theorem 1, we introduce the shorthand of

$$
\begin{aligned}
& \quad P=\min _{\boldsymbol{p}_{\mathrm{plc}}, \boldsymbol{p}_{\mathrm{vlc}}} P_{\mathrm{plc}}+P_{\mathrm{vlc}, 1} \\
& \text { s.t. }(7 c)-(7 e) .
\end{aligned}
$$

The problem of (7) is feasible only if $P_{T} \geq P$, where $P$ is the minimum power required by our constrained PLC-VLC network. Assume that the power $p_{\mathrm{vlc}, m}$ assigned to user $m$ 
relies on its achievable rate $R_{\mathrm{vlc}, m}$, where $R_{\mathrm{vlc}, m}>r_{m}$. Hence, the following relationship is obtained:

$$
2^{2 R_{\mathrm{vlc}, m} / B_{\mathrm{v}}}=1+\frac{\eta_{m} p_{\mathrm{vlc}, m}}{\eta_{m} P_{\mathrm{vlc}, m+1}+1}>2^{2 r_{m} / B_{\mathrm{v}}} .
$$

Accordingly, the power $p_{\mathrm{vlc}, m}$ allocated to user $m$ satisfies $p_{\mathrm{vlc}, m}>\left(2^{2 r_{m} / B_{\mathrm{v}}}-1\right)\left(\eta_{m} P_{\mathrm{vlc}, m+1}+1\right) / \eta_{m}$. This implies that the power $p_{\mathrm{vlc}, m}$ can be further reduced, so that $P$ is decreased. Hence, the power allocated to user $m$ can be reduced to:

$$
p_{\mathrm{vlc}, m}^{\prime}=\frac{2^{2 r_{m} / B_{\mathrm{v}}}-1}{\eta_{m}}\left(\eta_{m} P_{\mathrm{vlc}, m+1}+1\right)<p_{\mathrm{vlc}, m},
$$

when the achievable throughput is $R_{\text {vlc }, m}^{\prime}=r_{m}$. Meanwhile, for user $l \in\{1, \cdots, m-1\}$, the reduction of $p_{\mathrm{vlc}, m}$ leads to an increased $R_{\mathrm{vlc}, l}$. Similarly, the power $p_{\mathrm{vlc}, l}$ allocated to user $l$ can be sequentially reduced to $p_{\mathrm{vlc}, l}^{\prime}$, while still satisfying its minimum rate requirement. Hence, we infer that the constraint (7d) holds with equality for all users, while minimizing the total power. Using a recursion, we arrive at $P_{\mathrm{v}}=\min P_{\mathrm{vlc}, 1}=\sum_{m=1}^{M} 2^{\sum_{j=1}^{m-1} 2 r_{j} / B_{\mathrm{v}}}\left(2^{2 r_{m} / B_{\mathrm{v}}}-1\right) / \eta_{m}$, hence the problem in (17) can be rewritten as:

$$
\begin{gathered}
\quad P=\min _{\boldsymbol{p}_{\mathrm{plc}}} P_{\mathrm{plc}}+P_{\mathrm{v}} \\
\text { s.t. } R_{\mathrm{plc}} \geq \sum_{m} r_{m} ; \\
\boldsymbol{p}_{\mathrm{plc}} \geq \mathbf{0}_{N \times 1} .
\end{gathered}
$$

Since $P_{\mathrm{v}}$ has been found, we treat it as a constant. The Lagrangian function of (20) is given by:

$\mathcal{L}\left(\boldsymbol{p}_{\mathrm{plc}}, \xi\right)=\sum_{n} p_{\mathrm{plc}, n}-\xi\left[\varrho B \sum_{n} \log _{2}\left(1+p_{\mathrm{plc}, n} \lambda_{n}\right)-\sum_{m} r_{m}\right]$

where $\xi$ is the Lagrange multiplier. Taking the derivative of $\mathcal{L}\left(\boldsymbol{p}_{\mathrm{plc}}, \xi\right)$ with respect to $p_{\mathrm{plc}, n}$ and setting it to zero, the power assigned to the $n$th subcarrier in PLC is given by $p_{\mathrm{plc}, n}=\left(\frac{\xi \varrho B}{\ln 2}-\frac{1}{\lambda_{n}}\right)^{+}$, where $(x)^{+}=\max \{0, x\}$. Furthermore, this implies that $\xi>0$, otherwise the power of all subcarriers becomes negative. Therefore, by observing (20b), we get $\xi=\frac{\ln 2}{\varrho B} 2^{\left(\sum_{m} r_{m}-\varrho B \sum_{n} \log _{2} \lambda_{n}\right) / \varrho B_{\mathrm{p}}}$. Hence, having at least one feasible solution for (7) is guaranteed upon satisfying the condition in (8).

\section{REFERENCES}

[1] L. Chen, W. Wang, and C. Zhang, "Coalition formation for interference management in visible light communication networks," IEEE Trans. Veh. Technol., vol. 66, no. 8, pp. 7278-7285, Aug. 2017.

[2] H. Ma, L. Lampe, and S. Hranilovic, "Hybrid visible light and power line communication for indoor multiuser downlink," IEEE J. Opt. Commun. Netw., vol. 9, no. 8, pp. 635-647, Aug. 2017.

[3] N. Taherinejad, L. Lampe, and S. Mirabbasi, "An adaptive impedancematching system for vehicular power line communication," IEEE Trans. Veh. Technol., vol. 66, no. 2, pp. 927-940, Feb. 2017.

[4] M. Kashef, M. Abdallah, and N. Al-Dhahir, "Transmit power optimization for a hybrid PLC/VLC/RF communication system," IEEE Trans. Green Commun. Netw., vol. 2, no. 1, pp. 234-245, Mar. 2018.

[5] H. Marshoud, V. M. Kapinas, G. K. Karagiannidis, and S. Muhaidat, "Non-orthogonal multiple access for visible light communications," IEEE Photon. Technol. Lett., vol. 28, no. 1, pp. 51-54, Jan. 2016.

[6] J. Grubor, S. C. J. Lee, K. D. Langer, T. Koonen, and J. W. Walewski, "Wireless high-speed data transmission with phosphorescent white-light LEDs," in Proc. 33rd ECOC, pp. 1-2, Sep. 2007.
[7] Z. Ding, Z. Yang, P. Fan, and H. V. Poor, "On the performance of non-orthogonal multiple access in $5 \mathrm{G}$ systems with randomly deployed users," IEEE Signal Process. Lett., vol. 21, no. 12, pp. 1501-1505, Dec. 2014.

[8] S. S. Bawazir, P. C. Sofotasios, S. Muhaidat, and Y. Al-hammadi, "Multiple access for visible light communications: Research challenges and future trends," IEEE Access, vol. 6, pp. 26 167-26 174, Jun. 2018.

[9] R. Zhang, H. Claussen, H. Haas, and L. Hanzo, "Energy efficient visible light communications relying on amourphous cells," IEEE J. Sel. Areas Commun., vol. 34, no. 4, pp. 894-906, Apr. 2016.

[10] M. H. Rashid, Power electronics handbook, L. Reading, Ed. Hayton, J., 2017.

[11] M. Zimmermann and K. Dostert, "A multipath model for the powerline channel," IEEE Trans. Commun., vol. 50, no. 4, pp. 553-559, Apr. 2002.

[12] T. Esmailian, F. Kschischang, and P. Gulak, "In-building power lines as high-speed communication channels: Channel characterization and a test channel ensemble," Int. J. Commun. Syst., vol. 16, pp. 381-400, May 2003.

[13] M. Zimmermann and K. Dostert, "Analysis and modmodel of impulsive noise in broad-band powerline communications," IEEE Trans. Electromagn. Compat., vol. 44, no. 1, pp. 249-258, Feb. 2002.

[14] T. Komine and M. Nakagawa, "Fundamental analysis for visible-light communication system using LED lights," IEEE Trans. Consum. Electron., vol. 50, no. 1, pp. 100-107, Feb. 2004.

[15] A. Chaaban, Z. Rezki, and M. Alouini, "Fundamental limits of parallel optical wireless channels: Capacity results and outage formulation," IEEE Trans. Commun., vol. 65, no. 1, pp. 296-311, Jan. 2017.

[16] L. Yin, W. O. Popoola, X. Wu, and H. Haas, "Performance evaluation of non-orthogonal multiple access in visible light communication," IEEE Trans. Commun., vol. 64, no. 12, pp. 5162-5175, Dec. 2016.

[17] C. Chen, W. Zhong, H. Yang, and P. Du, "On the performance of MIMONOMA-based visible light communication systems," IEEE Photon. Technol. Lett., vol. 30, no. 4, pp. 307-310, Feb. 2018. 